\title{
Genetic Variations of Human Neuropsin Gene and Psychiatric Disorders: Polymorphism Screening and Possible Association with Bipolar Disorder and Cognitive Functions
}

\author{
Aiko Izumi' ${ }^{1,2}$, Yoshimi lijima', Hiroko Noguchi', Tadahiro Numakawa', Takeya Okada', Hiroaki Hori', \\ Tadafumi Kato $^{3}$, Masahiko Tatsumi ${ }^{4,5}$, Asako Kosuga ${ }^{4}$, Kunitoshi Kamijima ${ }^{4}$, Takashi Asada ${ }^{6}$, \\ Kunimasa Arima ${ }^{7}$, Osamu Saitoh $^{7}$, Sadao Shiosaka $^{2}$ and Hiroshi Kunugi*, \\ 'Department of Mental Disorder Research, National Institute of Neuroscience, National Center of Neurology and Psychiatry, Tokyo, Japan; \\ ${ }^{2}$ Division of Structural Cell Biology, Nara Institute of Science and Technology, Nara, Japan; ${ }^{3}$ Laboratory for Molecular Dynamics of Mental \\ Disorders, Brain Science Institute, RIKEN, Saitama, Japan; ${ }^{4}$ Department of Psychiatry, Showa University School of Medicine, Tokyo, Japan; \\ ${ }^{5}$ Yokohama Shinryo Clinic, Kanagawa, Japan; ${ }^{6}$ Department of Psychiatry, Institute of Clinical Medicine, University of Tsukuba, Ibaraki, Japan; \\ ${ }^{7}$ Department of Psychiatry, Musashi Hospital, National Center of Neurology and Psychiatry, Tokyo, Japan
}

\begin{abstract}
Human neuropsin (NP) (hNP) has been implicated in the progressive change of cognitive abilities during primate evolution. The hNP gene maps to chromosome $19 q / 3$, a region reportedly linked to schizophrenia and bipolar disorder. Therefore, hNP is a functional and positional candidate gene for association with schizophrenia, mood disorders, and cognitive ability. Polymorphism screening was performed for the entire hNP gene. The core promoter region was determined and whether or not transcriptional activity alters in an allele-dependent manner was examined by using the dual-luciferase system. Allelic and genotypic distributions of five single-nucleotide polymorphisms (SNPs) were compared between patients with schizophrenia $(n=439)$, major depression $(n=409)$, bipolar disorder $(n=207)$, and controls $(n=727)$. A possible association of the hNP genotype with memory index (assessed with Wechsler Memory Scale, revised, WMS-R) and intelligence quotient (IQ assessed with Wechsler Adult Intelligence Scale, revised; WAIS-R) was examined in healthy controls $(n=166)$. A total of 28 SNPs, including nine novel SNPs, were identified. No significant effects on transcriptional activity were observed for SNPs in the promoter region. A significant allelic association was found between several SNPs and bipolar disorder (for SNP23 at the 3' regulatory region; odds ratio 1.48, 95\% confidential interval I.16-1.88, P=0.00 I5). However, such an association was not detected for schizophrenia or depression. Significant differences were observed between SNP23 and attention/concentration sub-scale score of WMS-R $(P=0.016)$ and verbal IQ $(P<0.00 \mathrm{I})$. Genetic variation of the hNP gene may contribute to molecular mechanisms of bipolar disorder and some aspects of memory and intelligence.

Neuropsychopharmacology (2008) 33, 3237-3245; doi:I0.1038/npp.2008.29; published online 19 March 2008
\end{abstract}

Keywords: neuropsin; polymorphism screening; association study; bipolar disorder; memory; intelligence

\section{INTRODUCTION}

Neuropsin (NP, MIM: 605644), also called as kallikrein 8 (KLK8), is one of the secreted-type serine proteases, which was first cloned by our group in mice (Chen et al, 1995). NP mRNA is expressed specifically in the limbic system of mouse brain and is localized at the highest concentration in pyramidal neurons of the hippocampal CA1-3 sub-fields. Direct hippocampal stimulation and kindling induced by

\footnotetext{
*Correspondence: Dr H Kunugi, Department of Mental Disorder Research, National Institute of Neuroscience, National Center of Neurology and Psychiatry, 4-I-I, Ogawahigashi, Kodaira, Tokyo I878502, Japan, Tel/Fax: + 8142346 17|4, E-mail: hkunugi@ncnp.go.jp Received 7 September 2007; revised 10 January 2008; accepted 7 February 2008
}

amygdaloid stimulation caused a significant bilateral change in NP mRNA level in the hippocampal pyramidal neurons. The activity-dependent changes and the specific localization indicate that NP is involved in hippocampal plasticity (Chen et al, 1995). Indeed, NP has a regulatory effect on Schaffer-collateral at the early phase of long-term potentiation (LTP) (Komai et al, 2000). Mice lacking NP were significantly impaired in the Morris water maze and $\mathrm{Y}$ maze, suggesting that NP has an important role in learning and memory (Tamura et al, 2006). The human NP (hNP) gene was cloned by Yoshida et al (1998), and then localized to chromosome 19q13.3-q13.4 (Gan et al, 2000; Harvey et al, 2000). It consists of six exons and the first exon is nontranslational. Four alternative splicing variants have been identified (Mitsui et al, 1999; Magklara et al, 2001). The regular form is called type1, and type 2 contains a 135-bp 
insertion of $5^{\prime}$ upstream region of exon 3 (Mitsui et al, 1999). Interestingly, type 2 is a hominoid-specific splicing form (Li et al, 2004) and is expressed as abundantly as the type 1 in human brain (Mitsui et al, 1999). These findings points to the possibility that type $2 \mathrm{hNP}$ may contribute to progressive change of cognitive abilities during primate evolution. Moreover, dysfunctions in hNP may be involved in psychiatric diseases of cognitive abilities, including schizophrenia and mood disorders.

Family, twin, and adoption studies clearly suggest that genetic components play an important role in the pathogenesis of schizophrenia and mood disorders (reviewed by Shih et al, 2004). These psychiatric diseases demonstrate substantial cognitive deficits such as learning and memory (reviewed by Sharma and Antonova, 2003; Robinson et al, 2006; Green, 2006). A genome screen of linkage with bipolar disorder pedigrees provided evidence for susceptibility locus on chromosome 19q13 (Badenhop et al, 2002). Another genome scan in schizophrenia and bipolar pedigrees obtained an LOD ratio score of 1.5 at $19 \mathrm{q} 13$ in schizophrenic families (Macgregor et al, 2004). Therefore, the hNP gene is a good candidate gene for association with schizophrenia and mood disorders. Here we performed, for the first time, a polymorphism screening and association analysis of the hNP gene with schizophrenia, major depression, and bipolar disorder in a Japanese sample. A possible association of hNP with memory and intelligence in healthy subjects was also examined. In addition, we determined a core promoter region of the $\mathrm{hNP}$ gene and examined whether transcriptional activity varies in an allele-dependent manner.

\section{MATERIALS AND METHODS}

\section{Subjects}

Subjects for the association study were 439 patients with schizophrenia (240 males, mean age of 44.6 years (SD 14.0)), 409 patients with major depression (136 males, 53.3 years (15.9)), 207 patients with bipolar disorder (80 males, 50.2 years (14.7)), and 727 healthy controls (324 males, 43.5 years (16.4)). Among these, 104 patients with bipolar disorder and 108 controls were recruited around Shiga prefecture, approximately $350 \mathrm{~km}$ to the west of Tokyo, while the remaining 1570 subjects were recruited around Tokyo. Consensus diagnosis by at least two psychiatrists, one of whom was in charge of the patients, was made for each patient according to the Diagnostic and Statistical Manual of Mental Disorders, 4th edition (DSM-IV) criteria (American Psychiatric Association, 1994), on the basis of unstructured interviews and information from medical records. Control subjects were healthy volunteers who had no current or past contact to psychiatric services. Among them 213 controls were screened by the Japanese version of the Mini-International Neuropsychiatric Interview (Sheehan et al, 1998; Otsubo et al, 2005) by a research psychiatrist, whereas the remaining controls were not screened by such a structured interview. Participants were excluded if they had prior medical histories of central nervous system disease or severe head injury, or if they met the criteria for substance abuse or dependence, or mental retardation. All subjects were biologically unrelated Japanese. After description of the study, written informed consent was obtained from every subject. The study protocol was approved by institutional ethics committees.

\section{Neuropsychological Test Measures}

Among controls, 166 (53 males, 37.6 years (12.4)) were subject to memory and intelligence tests to detect possible association with the hNP genotype. These individuals were all screened by the Mini-International Neuropsychiatric Interview with respect to their psychiatric history and confirmed that they had no current or past history of psychiatric illness. To assess memory and intelligence, Japanese full versions of the Wechsler Memory ScaleRevised (WMS-R) (Sugishita, 2001; Wechsler, 1987) and the Wechsler Adult Intelligence Scale-Revised (WAIS-R) (WAIS-R, Shinagawa et al, 1990; Wechsler, 1981), respectively, were administered. Testing and scoring were performed by psychologists who were blind to genotypic data.

\section{Polymorphism Screening and Genotyping}

Venous blood was drawn from the subjects and genomic DNA was extracted from whole blood according to standard procedures. The genomic structure of hNP was determined from the National Center for Biotechnology Information (NCBI) (http://www.ncbi.nlm.nih.gov/) and the University of California at Santa Cruz (UCSC) database (http:// genome.ucsc.edu/cgi-bin/hgBlat). To screen for polymorphisms, we used direct sequencing with the Genome Lab-DTCS (Dye Terminator Cycle Sequencing) kit and CEQ8000 Genetic Analysis System (Beckman Coulter, Fullerton, CA). The entire 7428-bp genomic region containing all the exons, introns, the 1078-bp $5^{\prime}$ flanking region upstream to exon 1, and the 655-bp $3^{\prime}$ flanking region downstream to exon 6 were amplified from the genomic DNA of 24 randomly selected schizophrenic subjects. Sequences of 24 sets of primers for the polymorphism screening are listed in Supplementary Table S1.

The examined 7428-bp region seemed to constitute of single haplotype blocks (Supplementary Figure S1). We genotyped five single-nucleotide polymorphisms (SNPs) using TaqMan $5^{\prime}$-exonuclease allelic discrimination assay. They were A-807 $>$ G (ss73688625, SNP3), G-658 $>$ C (rs1722550, SNP4), IVS2-101T $>$ A (rs1701947, SNP6), IVS3-10A $>$ G (rs1701946, SNP7), and A5229 $>$ G (rs1612902, SNP23) (Figure 1). SNPs 3 and 4 were chosen from the $5^{\prime}$ regulatory region since they may have some effects on transcriptional activity, and SNPs 1, 2, 4, and 5 were in absolute linkage disequilibrium (LD) (ie, genotypes were completely the same) with each other. SNPs 6 and 7 were chosen because they were SNPs located close to the splicing sites of exon $3 \mathrm{a}$ (ie, an exon specific to type $2 \mathrm{hNP}$ ) and exon 4, respectively, and may have some effects on splicing. SNP23 was chosen from the $3^{\prime}$ region, since SNPs 15, 16, 17, 19, 21, 23, 24, 25, 27, and 28 were in absolute LD with each other. TaqMan probes and Universal PCR master mix were obtained from Applied Biosystems (Foster City, CA). Thermal cycling conditions for polymerase chain reaction (PCR) were 1 cycle at $95^{\circ} \mathrm{C}$ for $10 \mathrm{~min}$ followed by 50 cycles of $92^{\circ} \mathrm{C}$ for $15 \mathrm{~s}$ and $60^{\circ} \mathrm{C}$ for $1 \mathrm{~min}$. After 


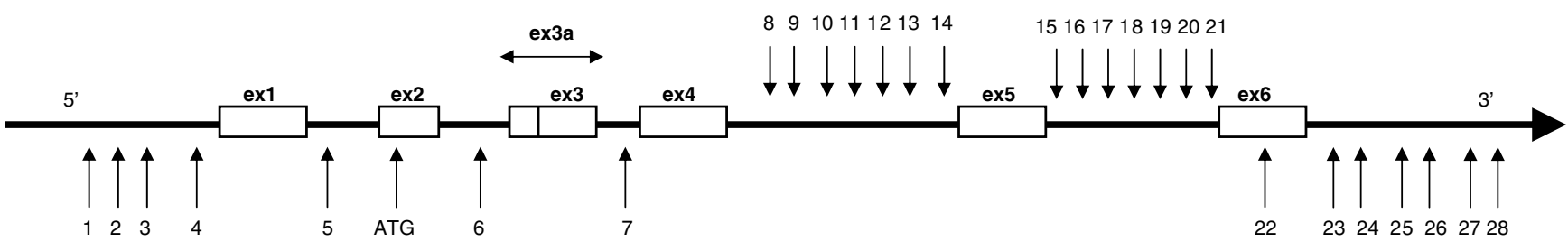

\begin{tabular}{|c|c|c|c|}
\hline 1. rs2569456 & g.G-1302>T & 15. rs1654539 & g. IVS5+106G>A \\
\hline 2. rs6509509 & g.C-948>T & 16. rs1722559 & g. IVS5+184C>T \\
\hline 3. ss73688625 & g.A-807>G & 17. rs1701914 & g. IVS5+187T>C \\
\hline 4. rs1722550 & g.G-658>C & 18. rs 1722560 & g. IVS5+673T>G \\
\hline 5. ss73688633 & g.IVS1+25,26 ins/del CA & 19. rs1722561 & g. IVS5+764T>C \\
\hline 6. rs1701947 & g.IVS2-236T>A (exon3) & 20. rs1701915 & g. IVS5-442A>G \\
\hline 7. rs1701946 & g.IVS3-10A $>G$ & 21. rs1627239 & g. IVS5-355G>T \\
\hline 8. ss73688628 & g.IVS4+596G>A & 22. ss73688626 & g. G5047>A (Val 286 lle) \\
\hline 9. rs7245714 & g.IVS4+866C>T & 23. rs1612902 & g. $A 5229>G$ \\
\hline 10. ss73688629 & g.IVS4+1034C>T & 24. rs1654542 & g. $T 5308>C$ \\
\hline 11. ss73688630 & g.IVS4-951A>G & 25. rs1654543 & g. $A 5435>G$ \\
\hline 12. rs12610313 & g.IVS4-624C>A & 26. ss73688627 & g.A5579>G \\
\hline 13. ss73688631 & g.IVS4-585C>T & 27. rs1897605 & g. T5646>C \\
\hline 14. ss73688632 & g.IVS4-306T>C & 28. rs1897606 & g. $C 5777>T$ \\
\hline
\end{tabular}

Figure I Genomic structure and identified polymorphisms in the human NP gene. A total of 28 SNPs, including one insertion/deletion (ins/del) polymorphism, were identified. The A of the translational start ATG is designated + I. Nine SNPs were novel and have been registered in the dbSNP (sstagged numbers). ex, exon; ex3a, exon3 in hNP type2.

amplification, the allele-specific fluorescence was measured with ABI PRISM 7900 Sequence Detection Systems (Applied Biosystems). Genotype data were read blind to the casecontrol status. Ambiguous genotype data were not included in the analysis.

\section{Promoter Assay in Primary Cultured Neurons}

Primary dissociated cultures were prepared from the brain cortex of postnatal 2-day-old rats (SLC, Shizuoka, Japan) as described previously (Numakawa et al, 2002). To generate plasmids for luciferase gene reporter assay, two differentially sized (964 and $128 \mathrm{bp}$ ) fragments of the $5^{\prime}$ flanking region of hNP were amplified by PCR with primers $5^{\prime}$-CGA CGCGTGCGTGTGCTGGGTTTGAA-3' (forward) and $5^{\prime}$-GA AGATCTCTAGAGCCTGGGGAGCTTCT- $3^{\prime}$ (reverse) for the 964-bp fragment, and $5^{\prime}$-CGACGCGTCCTCCTCTCCCTAGC CTCAG-3' (forward) and 5'-GAAGATCTCTAGAGCCTGGG GAGCTTCT $3^{\prime}$ (reverse) for the 128-bp fragment. These primers were designed to incorporate $M l u \mathrm{I}$ (forward) and BglII (reverse) restriction sites, and the PCR product was inserted into the multiple cloning site upstream of the luciferase coding region in the pGL3-Basic vector (Promega, Madison, WI). The inserted sequence was confirmed with the auto sequencer CEQ8000 in both directions using primers $5^{\prime}$-TCTCCATCAAAACAAAACGAA- $3^{\prime}$ and $5^{\prime}$-TTCC ATCTTCCAGCGGATA- $3^{\prime}$.

Among the four SNPs (SNPs 1-4; see Figure 1) in the $5^{\prime}$ upstream region (ie, putative promoter region) of the hNP gene, the genotypes of SNPs 1, 2, and 4 were completely the same for all the 24 schizophrenic subjects, and we found a significant association of bipolar disorder with SNP4 but not SNP3 (see results). In addition, haplotypes containing the A allele (the major allele), but not the G-allele, of SNP3 showed some evidence for association with bipolar disorder in haplotype analysis (see Table 2).
We therefore made two allele-specific promoter fragments (haplotypes consisting of SNPs 1-2-3-4 were G-C-A-G and T-T-A-C) of 964- and 128 bp upstream from the transcription initiation site, which were subject to the luciferase reporter gene assay. The plasmid constructs were transfected into cultured neurons at 5 days in vitro. Cells on 24-well plates were co-transfected with $3200 \mathrm{ng}$ of pGL3Basic firefly luciferase reporter vectors, which included allele-specific promoter fragments of 964 and $128 \mathrm{bp}$, and $100 \mathrm{ng}$ of phRL-TK Renilla luciferase vector (Promega, Tokyo, Japan) as an internal control using Lipofectamine 2000 regent (Invitrogen, Tokyo, Japan). As negative control, an empty pGL3-Basic vector was simultaneously transfected in all the experiments. At $24 \mathrm{~h}$ after transfection, luciferase activity was measured using a Dual-Luciferase Reporter Assay System (Promega) and a Lumat LB 9507 luminometer (Berthold, Bad Wildbad, Germany), as described previously (Tadokoro et al, 2004; Okada et al, 2006). Firefly and Renilla luciferase activities were quantified sequentially as relative light unit (RLU) by addition of their respective substrates according to the protocol of the supplier. The ratio of firefly RLU to Renilla RLU of each sample was automatically computed. The activity of each construct was expressed at the relative value compared with that of pGL3-Promoter (as a positive control), and these relative values were computed by $t$-test. Primary cultured cells were prepared six times and transfection was performed quadruplicate for each cell culture.

\section{Statistical Analysis}

Deviations of genotype distributions from Hardy-Weinberg equilibrium were assessed with $\chi^{2}$-test for goodness of fit. Genotype and allele distributions of each SNP were compared between patients and controls using $\chi^{2}$-test for independence. The association of the hNP genotype with 
memory and intelligence was examined by multiple analysis of variance (MANOVA) controlling for possible confounders (age, sex, and education years). These tests were performed with the SPSS software version 11 (SPSS Japan, Tokyo, Japan). The LD $\left(D^{\prime}\right)$ between polymorphisms was examined using the Haploview program (http://www. broad.mit.edu/mpg/haploview/) (Barrett et al, 2005) and haplotype-based association analyses were performed with COCAPHASE software version 2.4 (http://www.mrc-bsu. cam.ac.uk/personal/frank/software/unphased/; Dudbridge et al, 2000). The expectation-maximization (EM) and 'droprare' options were used. Haplotypes with frequencies less than $3 \%$ were considered to be rare. We examined associations by permutation procedure (10000 replications) to determine the empirical significance. All $P$-values reported are two-tailed. Statistical significance was considered when $P<0.05$.

\section{RESULTS}

\section{Polymorphism Identification and Genotyping}

The 7428-bp genomic region containing all the exons, introns, $5^{\prime}$ flanking, and $3^{\prime}$ flanking regions of hNP were screened for polymorphisms in 24 schizophrenic patients. A total of 28 SNPs, including one insertion/deletion (ins/del) polymorphism, were identified (Figure 1). Among them 19 SNPs had already been listed in the NCBI dbSNP database, whereas nine SNPs were novel. Four SNPs were located in $5^{\prime}$ upstream, one SNP in exon 6, six SNPs in $3^{\prime}$ downstream, and the remaining 17 polymorphisms in introns. There was only one SNP that resulted in an amino-acid change, SNP22 (G5047 > A; Val286Ile: the number of the amino acid is according to NP_653088), which gave rise to a restriction site for AcyI and was located at an evolutionarily conserved (rodents through humans) residue. This non-synonymous polymorphism was found in only one schizophrenic patient. Additional genotyping was performed for 178 individuals with schizophrenia; however, there was no individual carrying the 286Ile allele, indicating that this amino-acid change is a rare mutation. The LD between SNPs is shown in Supplementary Figure S1, indicating that the entire genomic region consists of single haplotype block. Genotypes for SNPs 1, 2, 4, and 5, those for SNPs 8 and 9, those for SNPs 10 and 13, and those for SNPs15, 16, 17, 19, 21, 23, $24,25,27$, and 28 , respectively, were completely the same as each other for the 24 individuals.

\section{Promoter Assay}

We identified four SNPs in the 1078-bp $5^{\prime}$ upstream region of the hNP gene, and SNPs 1, 2, and 4 were found to be associated with bipolar disorder, memory, and intelligence quotient (IQ) (see below). Furthermore, to our knowledge, there is no information in the literature on the location of core promoter of the hNP gene. We therefore performed a promoter assay using the dual-luciferase system (Promega) in rat cultured cortical neurons and examined whether transcriptional activity alters in an allele-dependent manner. As shown in Figure 2a, pGL3-Basic vectors containing 128- and 964-bp fragments, which consisted of major alleles for the four SNPs, demonstrated substantially higher RLEs
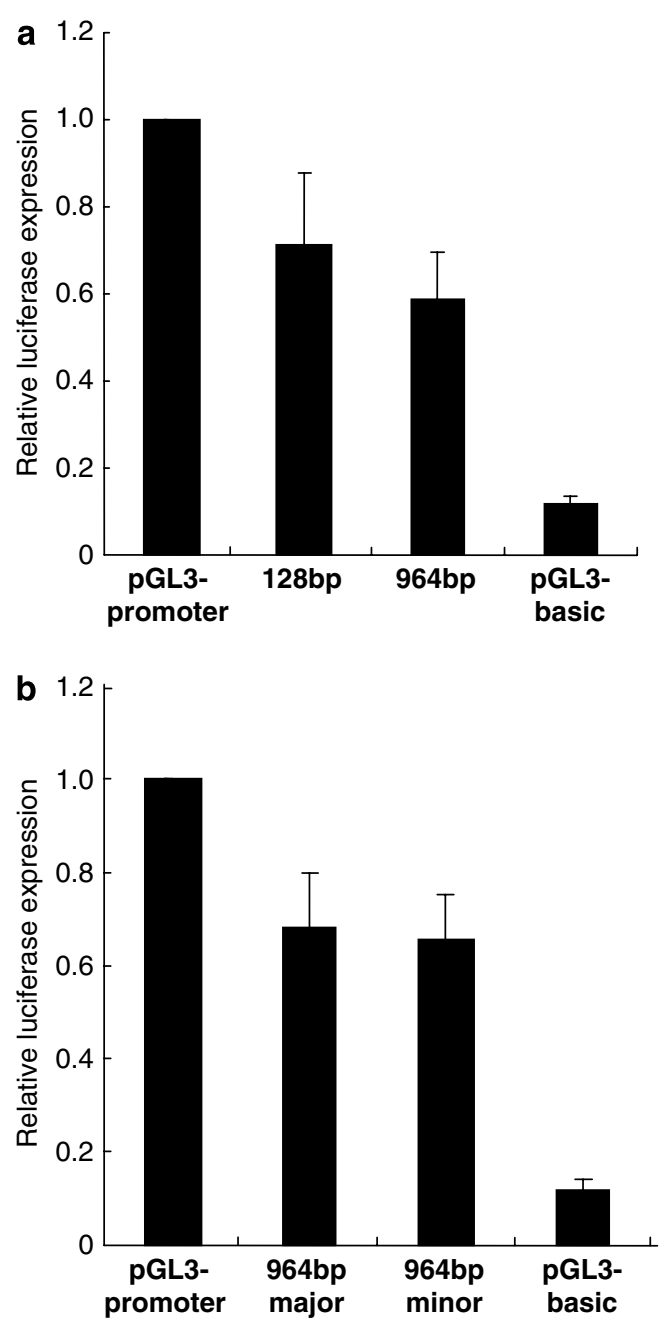

Figure 2 Promoter assay. (a) RLE for pGL3-Basic vector with insertion of 128 and 964 bp of the hNP 5' flanking regions in comparison with pGL3Basic vector, which dose not contain a promoter sequence. The RLE for pGL3-promoter vector containing SV40 promoter (positive control vector) was assigned a value of I. Both 128- and 964-bp fragments showed substantially higher RLE compared with pGL3-basic vector without promoter sequence. (b) Comparison of RLE between the major (G-CA-G for SNPs I-2-3-4) and minor (T-T-A-C) alleles. No significant difference was found between the two alleles.

(relative luciferase expression) than that of pGL3-basic empty vector, suggesting that the core promoter region is located within the 128-bp fragment. We then cloned the 964-bp allele-specific promoter fragments (SNP1-2-3-4; major allele: G-C-A-G, minor allele: T-T-A-C) and compared RLEs between the two alleles (Figure 2b); however, we found no significant difference in the RLE between the two haplotype fragments. These results suggest that SNPs 1, 2, and 4 might not influence the transcriptional activity of the hNP gene.

\section{Association with Psychiatric Diseases}

We genotyped five SNPs (SNPs 3, 4, 6, 7, and 23) to examine possible association with schizophrenia, major depression, and bipolar disorder. Genotype and allele distributions in the diagnostic groups are shown in Table 1. Genotype 
Table I Genotype and Allele Distributions of the five SNPs of the hNP Gene in Patients with Schizophrenia, those with Major Depression, those with Bipolar Disorder, and the Controls

\begin{tabular}{|c|c|c|c|c|c|c|c|c|c|c|c|}
\hline \multirow[t]{2}{*}{ SNP } & \multirow[t]{2}{*}{ Diagnosis } & \multirow[t]{2}{*}{$N$} & \multicolumn{3}{|c|}{$\begin{array}{c}\text { Genotype } \\
\text { frequency (GF) }\end{array}$} & \multicolumn{2}{|c|}{$\begin{array}{l}\text { Allele frequency } \\
\text { (AF) }\end{array}$} & \multirow[t]{2}{*}{$\begin{array}{l}\text { Odds ratio } \\
(95 \% \mathrm{Cl})\end{array}$} & \multicolumn{3}{|c|}{$\chi^{2}$-Test vs controls } \\
\hline & & & & & & & & & $\begin{array}{l}\text { GF vs } \\
\text { HW }\end{array}$ & $G F(d f=2)$ & $A F(d f=I)$ \\
\hline \multirow[t]{3}{*}{ SNP3 } & & & $\mathrm{A} / \mathrm{A}$ & $A / G$ & $\mathrm{G} / \mathrm{G}$ & $A$ & G & & & & \\
\hline & Controls & 696 & $462(0.66)$ & $208(0.30)$ & $26(0.04)$ & I I $32(0.81)$ & $260(0.19)$ & & 0.67 & & \\
\hline & $\mathrm{BD}$ & 202 & $139(0.69)$ & $56(0.28)$ & $7(0.03)$ & $334(0.83)$ & $70(0.17)$ & $1.09(0.82-1.46)$ & 0.65 & $\chi^{2}=0.4 P=0.81$ & $\chi^{2}=0.36 P=0.54$ \\
\hline
\end{tabular}

SNP4

Controls $\quad 683 \quad 388(0.57) \quad 243(0.36) \quad 52(0.08) \quad 1019(0.75) \quad 347(0.25)$

SZ $\quad 406 \quad 234(0.58) \quad 150(0.37) \quad 22(0.05) \quad 618(0.76) \quad 194(0.24)$

0.11

$\chi^{2}=1.97 P=0.37 \quad \chi^{2}=0.62 P=0.43$

$\mathrm{BD}$

$19891(0.46) \quad 90(0.45) \quad 17(0.09)$

$272(0.69) \quad 124(0.31) \quad 1.33(1.04-1.7)$

0.43

$\chi^{2}=0.5 P=0.78$

$\chi^{2}=0.58 P=0.47$

SNP6

$\begin{array}{lcccc} & & T / T & T / A & \text { A/A } \\ \text { Controls } & 711 & 316(0.44) & 306(0.43) & 89(0.13) \\ \text { SZ } & 422 & 195(0.46) & 192(0.45) & 35(0.08) \\ \text { MD } & 378 & 171(0.45) & 164(0.43) & 43(0.11) \\ \text { BD } & 197 & 70(0.36) & 99(0.50) & 28(0.14)\end{array}$

$\begin{array}{ccc}T & A\end{array}$

$938(0.66) \quad 484(0.34)$

0.27

$\begin{array}{lllll}582(0.69) & 262(0.3 I) & \text { I.I7 (0.97-I.4I) } & 0.20\end{array}$

$506(0.67) \quad 250(0.33) \quad 1.06(0.88-1.29) \quad 0.70$

$\chi^{2}=4.86 P=0.09$

$\chi^{2}=2.15 P=0.14$

$239(0.6 \mid) \quad$ I $55(0.39) \quad$ I.25 (0.99-।.58) $\quad 0.46$

$\chi^{2}=0.31 P=0.86$

$\chi^{2}=0.21 P=0.65$

$\chi^{2}=5.03 P=0.08$

$\chi^{2}=3.8 P=0.051$

SNP7

Controls

A/A $\quad A / G \quad G / G$

A G

SZ

$718 \quad 325(0.45) \quad 314(0.44) \quad 79(0.11)$

$964(0.67) \quad 472(0.33)$

$433209(0.48) \quad 190(0.44) \quad 34(0.08)$

$608(0.70) \quad 258(0.30) \quad 1.16(0.96-1.40)$

0.81

$\begin{array}{llllll}M D & 387 & 182(0.47) & 163(0.42) & 42(0.11)\end{array}$

$527(0.68) \quad 247(0.32) \quad 1.05(0.87-1.28) \quad 0.55$

$\chi^{2}=3.26 P=0.20$

$\chi^{2}=2.36 P=0.12$

$\mathrm{BD}$

$203 \quad 72(0.35) \quad 103(0.51) \quad 28(0.14)$

$247(0.61) \quad 159(0.39) \quad 1.31(1.04-1.65) \quad 0.36$

$\chi^{2}=0.33 P=0.85$

$\chi^{2}=0.21 P=0.65$

SNP23

Controls
SZ
MD
BD

$\begin{array}{rllll} & \text { A/A } & \text { A } / G & \text { G/G } & A \\ 714 & 428(0.60) & 241(0.34) & 45(0.06) & 1097 \\ 421 & 267(0.63) & 135(0.32) & 19(0.05) & 669 \\ 388 & 240(0.62) & 127(0.33) & 21(0.05) & 607 \\ 204 & 98(0.48) & 86(0.42) & 20(0.10) & 282\end{array}$

A G

$1097(0.77) \quad 331(0.23)$

0.16

$\begin{array}{lllll}669 & (0.79) \quad 173(0.21) & 1.17(0.94-1.44) & 0.71\end{array}$

$607(0.78) \quad 169(0.22) \quad 1.08(0.87-1.34) \quad 0.44$

$\chi^{2}=2.25 P=0.32$

$\chi^{2}=2.13 P=0.14$

$282(0.69) \quad 126(0.31) \quad 1.48(1.16-1.88)$

0.86

$\chi^{2}=0.56 P=0.75$

$\chi^{2}=0.56 P=0.45$

Abbreviations: 95\% Cl, 95\% confidence interval; BD, bipolar disorder; df, degrees of freedom; hNP, human neuropsin; HW, Hardy-Weinberg; MD, major depression; SNP, single-nucleotide polymorphism; SZ, schizophrenia.Significant p-values are gray colored.

distributions of these SNPs did not deviate significantly from Hardy-Weinberg equilibrium, except for SNP3 in patients with major depression $(P=0.02)$. There was no significant difference in genotype or allele distribution for any SNP between patients and controls for schizophrenia or major depression. However, there was a significant difference in genotype distributions between patients with bipolar disorder and controls for three SNPs, that is, SNPs 4,7 , and 23. Allele frequencies for these SNPs also differed significantly between the two groups. $P$-values, odds ratios, and their $95 \%$ confidence interval (CI) are shown in Table 1. Then we performed haplotype-based analysis with a twomarker sliding window method. We obtained no evidence of a significant association for schizophrenia or major depression (data not shown). With respect to bipolar disorder, we obtained significant individual $P$-values for all combinations of two markers; however, significant global
$P$-value (0.0068) was obtained only when haplotype consisted of SNPs 7 and 23 (Table 2). Furthermore, overall global $P$-value $(P=0.083)$, considering all multiple testing for all the combinations of two-marker haplotypes, just failed to reach statistical significance. Thus, we did not obtain any stronger evidence for association in the haplotype-based analysis than in the single-marker analysis of SNP23 $(P=0.0015)$.

\section{Association with Memory and IQ}

Among the 166 controls whose memory scale and IQ were measured, SNP23 (A/G) was successfully genotyped in 163 individuals. Mean (SD) index scores of verbal memory, visual memory, general memory, attention and concentration, and delayed recall in the 163 controls were 110.9 (13.7), 109.9 (9.0), 112.2 (12.1), 103.9 (13.5), and 112.1 
Table 2 Two-Marker Haplotype Analysis in Patients with Bipolar Disorder and Controls.

\begin{tabular}{|c|c|c|c|c|c|c|c|c|c|}
\hline \multicolumn{5}{|c|}{ Markers } & \multicolumn{2}{|c|}{ Haplotype frequency } & \multirow[b]{2}{*}{ Individual } & \multicolumn{2}{|c|}{$P$-value } \\
\hline SNP3 & SNP4 & SNP6 & SNP7 & SNP23 & BD & Controls & & Global $^{\mathbf{a}}$ & Overall global $^{a}$ \\
\hline \multirow{2}{*}{ A } & C & $A$ & & & 0.30 & 0.24 & 0.030 & 0.10 & \\
\hline & & $A$ & G & & 0.39 & 0.33 & 0.028 & 0.11 & \\
\hline
\end{tabular}

Abbreviations: BD, bipolar disorder; SNP, single-nucleotide polymorphism.

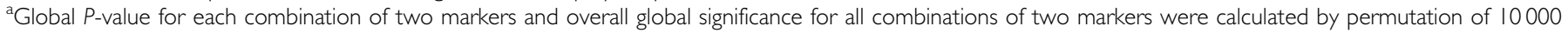
simulations.

(12.0), respectively. Mean (SD) full-scale IQ, verbal IQ, and performance IQ were 109.3 (11.6), 107.3 (12.9), and 110.3 (11.7), respectively. Since SNP23 showed the strongest association with bipolar disorder (G-allele was the risk allele) among the 5 SNPs examined, memory and IQ were compared between those who carried the G-allele (carrier, $\mathrm{G} / \mathrm{G}$ or $\mathrm{A} / \mathrm{G}, N=64$ ) and those who did not (non-carrier, $\mathrm{A} / \mathrm{A}, N=99$ ) (Figure 3 ). Since the number of individuals with $\mathrm{G} / \mathrm{G}$ genotype was very small $(N=8)$, they were combined with those with the A/G genotype. With respect to sub-scales of WMS-R, the mean score of attention/ concentration was significantly lower in carriers than in noncarriers $(P=0.016)$; however, there were no significant differences between the two groups for the remaining sub-scales (verbal memory, visual memory, general memory, and delayed recall). With respect to WAIS-R, there was a significant difference in full-scale IQ $(P=0.018)$ between the two groups. When verbal and performance IQ were examined separately, there was a highly significant difference in verbal IQ $(P<0.001)$, but not in performance IQ, between the two groups. The mean verbal IQ (SD) for carriers and non-carriers was 103.4 (12.9) and 109.8 (12.3), respectively. As for the other SNPs, similar results are obtained (data not shown) because of the tight LD across the SNPs.

\section{DISCUSSION}

In the present study, we performed polymorphism screening and identified 28 SNPs, including nine novel SNPs, in the 7428-bp region of the whole hNP gene, including the $5^{\prime}$ and $3^{\prime}$ flanking regions. Then we performed promoter assay and determined a core promoter region of the hNP gene, although failing to find significant effects of SNPs on transcriptional activity. Association analysis using five SNPs as markers revealed significant difference in genotype and allele distributions for some of the SNPs between patients and controls for bipolar disorder, but not for schizophrenia or major depression. When a possible association of the SNPs with memory and IQ was examined in healthy control subjects, we found significant differences in attention/ concentration sub-scale score of the WMS-R and verbal IQ between genotypes.

Among the 28 SNPs identified, there was only one SNP in the exons; SNP22 was a Val286Ile missense mutation in exon 6 , which was detected in a patient with schizophrenia. Additional genotyping for 178 schizophrenic subjects did not find anyone carrying this variant, indicating that this is a rare mutation. Thus, whether this mutation is pathogenic or not is unclear. Since we examined only 24 individuals for polymorphism screening, we may have missed some rare mutations as the SNP22.

Our promoter assay in rat primary cultured neurons suggested that the core promoter is present in the 128-bp $5^{\prime}$ upstream region of the hNP gene. Since the RLE of pGL3vector containing the 964-bp fragment was somewhat lower than that of pGL3-vector containing 128-bp fragment, a silencer-like region may be present between 128- and 964bp positions upstream of the hNP gene. Then we examined whether transcriptional activity differs in an allele-dependent manner; however, we found no significant difference between alleles. These results suggest that SNPs 1, 2, and 4 might not influence the transcriptional activity of the hNP gene. According to the TFSEARCH database (http:// mbs.cbrc.jp/research/db/TFSEARCHJ.html), these SNPs are not located on any of the binding sites of transcriptional factors, which is in line with our finding of no significant difference between the alleles.

In our association study with psychiatric diseases, we found, for the first time, significant differences in genotype and allele distributions between patients with bipolar disorder and controls. The best $P$-value was obtained for SNP23 in allele distribution $(P=0.0015$, odds ratio 1.48 , 95\% CI 1.16-1.88). This $P$-value remained significant even after correcting the critical $P$-value for Bonferroni's multiple testing (15 comparisons: 5 SNPs $\times 3$ diseases). Haplotypebased analysis also yielded nominally significant results particularly when SNP23 was included in markers of analysis. These results suggest that SNP23 or other unknown SNPs in LD with SNP23 confers susceptibility to bipolar disorder. Since hNP is a part of a gene cluster (kallikreins), there remains a possibility that variations of some other kallikrein gene might be truly responsible to giving susceptibility to bipolar disorder. The results are in line with a previous study reporting a susceptibility locus for bipolar disorder on chromosome 19q13 (Badenhop et al, 2002). A possible limitation is that a portion of patients with bipolar disorder and controls were recruited in a geographically different area (ie, Shiga prefecture but not in Tokyo), which may have resulted in a population stratification; however, the minor allele frequency of SNP23 was very similar in controls from Shiga and those from Tokyo $(0.233$ in Shiga and 0.232 in Tokyo), suggesting that the effect of stratification is unlikely. Another limitation might be that 

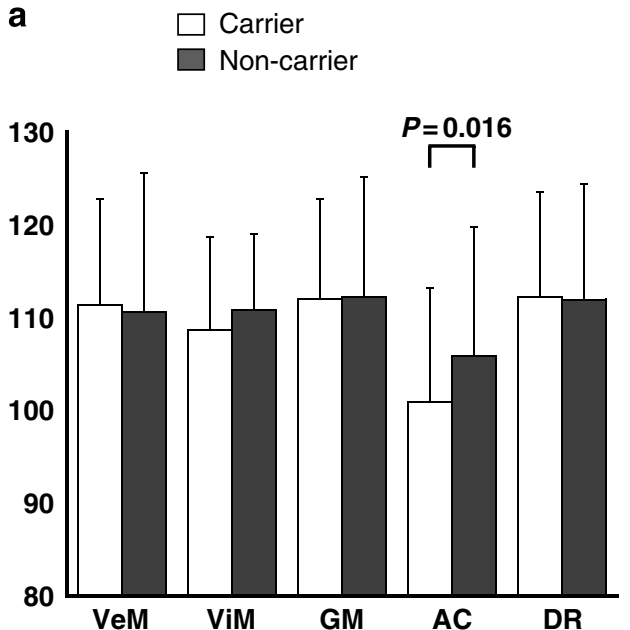

b

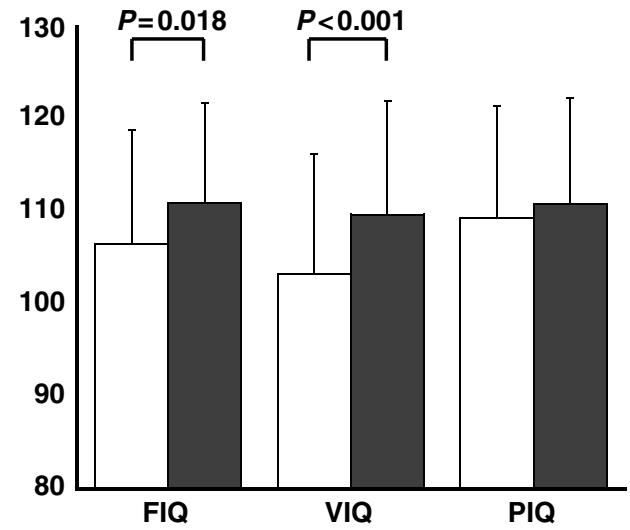

Figure 3 Relationship of memory and IQ with genotype of SNP23. Memory and IQ were compared between those who carried the G allele (carrier: $\mathrm{G} / \mathrm{G}$ or A/G) of SNP23 and those who did not (non-carrier: A/A). (a) Memory and genotype. VeM, verbal memory; ViM, visual memory; GM, general memory; AC, attention and concentration; DR, delayed recall. (b) IQ and genotype. FIQ, full-scale IQ; VIQ, verbal IQ; PIQ, performance IQ.

we did not conduct structured interview for diagnosis of the patients. However, consensus diagnosis was made by at least two psychiatrists one of whom was in charge of the patients; thus, the possibility of misdiagnosis might be minimal. In addition, our sample size (207 bipolar disorder subjects and 727 controls) was not very large, and thus further investigations in other samples are required to draw any conclusion. With respect to schizophrenia or major depression, we did not obtain any evidence for association with hNP.

Interestingly, we found significant association of memory and IQ with the hNP gene in healthy subjects. Carrying the G-allele of SNP23, the risk allele for bipolar disorder, was associated with lower score in attention/concentration assessed with the WMS-R $(P=0.016)$ and lower verbal IQ assessed with WAIS-R $(P<0.001)$. The evidence for the former association (with attention/concentration) was weak and it would not be significant any more after correcting for multiple testing; however, the latter association (with verbal IQ) was highly significant and remained significant even when multiple testing was taken into consideration. Since bipolar disorder shows a wide range of cognitive deficits, including memory and IQ (Schretlen et al, 2007; Daban et al, 2006), the observed impact on intelligence may have some relevance to susceptibility to bipolar disorder. However, given that deficits in intelligence and memory are generally worse in schizophrenia than in bipolar disorder, alterations in hNP may have some effects specific to molecular mechanisms of bipolar disorder.

$\mathrm{NP}$ is a secretory serine protease that degrades cell adhesion molecule L1 (CAM-L1) (Matsumoto-Miyai et al, 2003) and is possibly involved in the synaptogenesis and maturation of orphan and small synapses (Nakamura et al, 2006). Furthermore, NP has been shown to be involved in activity-dependent synaptic plasticity, that is, LTP and kindling epileptogenesis (Komai et al, 2000; Okabe et al, 1996). As mentioned above, the type 2 splice variant has been shown to be expressed as abundant as the type 1 in human brain (Mitsui et al, 1999) and the hominoid-specific form (Li et al, 2004), which occurred through a humanspecific T-to-A mutation (c.71-127T $>$ A) during primate evolution (Lu et al, 2007). Taken together, NP is involved in synaptic plasticity via modulation of synaptic structure, and may play an important role in brain function of higher order such as learning, memory, and mental disorders. With respect to psychiatric diseases, indeed, altered expression levels of CAM-L1 mRNA and protein have been reported in postmortem brains of depressed patients (Laifenfeld et al, 2005). In line with this, chronic antidepressants increase expression levels of CAM-LI in rats (Sairanen et al, 2007; Laifenfeld et al, 2002). It would be intriguing to examine the expression levels of NP in postmortem brains of psychiatric patients.

We found that SNP23 is most associated with bipolar disorder among the examined SNPs. Haplotype-based analysis did not yield any stronger results, suggesting that SNP23 may be responsible for giving susceptibility to bipolar disorder. In addition, SNP23 showed strong impact on verbal IQ in healthy subjects. SNP23 is located $69 \mathrm{bp}$ downstream to the $3^{\prime}$ end of exon 6 (the final exon). Thus SNP23 is on the $3^{\prime}$ regulatory region of the hNP gene. Growing evidence has shown that $3^{\prime}$ regulatory regions of human genes play an important role in regulating mRNA $3^{\prime}$ end formation, stability/degradation, nuclear export, subcellular localization, and translation, and are consequently rich in regulatory elements. Indeed, several diseases have been reported to be associated with variants in the $3^{\prime}$ regulatory region (Chen et al, 2006). Notably, the major allele (A) of SNP23 differs from the corresponding base (G) in monkeys or apes (ie, rhesus macaques or chimpanzees), according to the UCSC database, and thus it is not evolutionally conserved. It is interesting that carriers of the $\mathrm{G}$ allele were found to be poorer in memory and IQ subscales than individuals with A/A genotype in the present study. Although SNP23 is not located on obvious motifs or conserved sequence elements, it is also possible that this human-specific mutation may contribute to the higher memory and intelligence functions in humans. If our results 
are replicated in other samples, it is important to elucidate the possible functional effects of SNP23 on regulation of hNP mRNA, which may contribute to understanding of the pathogenesis of bipolar disorder and brain function of higher order specific to human beings.

\section{ACKNOWLEDGEMENTS}

This study was supported by Health and Labor Sciences Research Grants (Research on Psychiatric and Neurological Diseases and Mental Health; Research on Human Genome and Tailor-made medicine), the Program for Promotion of Fundamental Studies in Health Sciences of the National Institute of Biomedical Innovation (NIBIO), Japan Health Sciences Foundation (Research on Health Sciences focusing on Drug innovation), Grant-in-Aid for Scientific Research on Priority Areas of Applied Genomics from the Ministry of Education, Culture, Sports, Science and Technology of Japan. We thank Ms Tomoko Shizuno for technical assistance.

\section{DISCLOSURE/CONFLICT OF INTEREST}

All authors declare that they have no conflict of interests to disclose.

\section{REFERENCES}

American Psychiatric Association (1994). Diagnostic and Statistical Manual of Mental Disorders, 4th edn. American Psychiatric Press: Washington, DC.

Badenhop RF, Moses MJ, Scimone A, Mitchell PB, Ewen-White KR, Rosso A et al (2002). A genome screen of 13 bipolar affective disorder pedigrees provides evidence for susceptibility loci on chromosome 3 as well as chromosomes 9, 13 and 19. Mol Psychiatry 7: 851-859.

Barrett JC, Fry B, Maller J, Daly MJ (2005). Haploview: analysis and visualization of $\mathrm{LD}$ and haplotype maps. Bioinformatics 21: 263-265.

Chen JM, Férec C, Cooper DN (2006). A systematic analysis of disease-associated variants in the $3^{\prime}$ regulatory regions of human protein-coding genes I: general principles and overview. Hum Genet 120: 1-21.

Chen ZL, Yoshida S, Kato K, Momota Y, Suzuki J, Tanaka T et al (1995). Expression and activity-dependent changes of a novel limbic-serine protease gene in the hippocampus. J Neurosci 15: 5088-5097.

Daban C, Martinez-Aran A, Torrent C, Tabarés-Seisdedos R, Balanzá-Martínez V, Salazar-Fraile J et al (2006). Specificity of cognitive deficits in bipolar disorder versus schizophrenia. A systematic review. Psychother Psychosom 75: 72-84.

Dudbridge F, Koeleman BP, Todd JA, Clayton DG (2000). Unbiased application of the transmission/disequilibrium test to multilocus haplotypes. Am J Hum Genet 66: 2009-2012.

Gan L, Lee I, Smith R, Argonza-Barrett R, Lei H, McCuaig J et al (2000). Sequencing and expression analysis of the serine protease gene cluster located in chromosome 19q13 region. Gene 257: 119-130.

Green MF (2006). Cognitive impairment and functional outcome in schizophrenia and bipolar disorder. J Clin Psychiatry 67: 3-8.

Harvey TJ, Hooper JD, Myers SA, Stephenson SA, Ashworth LK, Clements JA (2000). Tissue-specific expression patterns and fine mapping of the human kallikrein (KLK) locus on proximal 19q13.4. J Biol Chem 275: 37397-37406.
Komai S, Matsuyama T, Matsumoto K, Kato K, Kobayashi M, Imamura $\mathrm{K}$ et al (2000). Neuropsin regulates an early phase of Schaffer-collateral long-term potentiation in the murine hippocampus. Eur J Neurosci 12: 1479-1486.

Laifenfeld D, Karry R, Klein E, Ben-Shachar D (2005). Alterations in cell adhesion molecule L1 and functionally related genes in major depression: a postmortem study. Biol Psychiatry 57: 716-725.

Laifenfeld D, Klein E, Ben-Shachar D (2002). Norepinephrine alters the expression of genes involved in neuronal sprouting and differentiation: relevance for major depression and antidepressant mechanisms. J Neurochem 83: 1054-1064.

Li Y, Qian YP, Yu XJ, Wang YQ, Dong DG, Sun W et al (2004). Recent origin of a hominoid-specific splice form of neuropsin, a gene involved in learning and memory. Mol Biol Evol 21: 2111-2115.

Lu ZX, Peng J, Su B (2007). A human-specific mutation leads to the origin of a novel splice form of neuropsin (KLK8), a gene involved in learning and memory. Hum Mutat 28: 978-984.

Macgregor S, Visscher PM, Knott SA, Thomson P, Porteous DJ, Millar JK et al (2004). A genome scan and follow-up study identify a bipolar disorder susceptibility locus on chromosome 1q42. Mol Psychiatry 9: 1083-1090.

Magklara A, Scorilas A, Katsaros D, Massobrio M, Yousef GM, Fracchioli $S$ et al (2001). The human KLK8 (neuropsin/ovasin) gene: identification of two novel splice variants and its prognostic value in ovarian cancer. Clin Cancer Res 7: 806-811.

Matsumoto-Miyai K, Ninomiya A, Yamasaki H, Tamura H, Nakamura Y, Shiosaka S (2003). NMDA-dependent proteolysis of presynaptic adhesion molecule L1 in the hippocampus by neuropsin. J Neurosci 23: 7727-7736.

Mitsui S, Tsuruoka N, Yamashiro K, Nakazato H, Yamaguchi N (1999). A novel form of human neuropsin, a brain-related serine protease, is generated by alternative splicing and is expressed preferentially in human adult brain. Eur J Biochem 260: 627-634.

Nakamura Y, Tamura H, Horinouchi K, Shiosaka S (2006). Role of neuropsin in formation and maturation of Schaffer-collateral L1cam-immunoreactive synaptic boutons. J Cell Sci 119: 1341-1349.

Numakawa T, Yamagishi S, Adachi N, Matsumoto T, Yokomaku D, Yamada $\mathrm{M}$ et al (2002). Brain-derived neurotrophic factorinduced potentiation of $\mathrm{Ca}(2+)$ oscillations in developing cortical neurons. J Biol Chem 277: 6520-6529.

Okabe A, Momota Y, Yoshida S, Hirata A, Ito J, Nishino H et al (1996). Kindling induces neuropsin mRNA in the mouse brain. Brain Res 728: 116-120.

Okada T, Hashimoto R, Numakawa T, Iijima Y, Kosuga A, Tatsumi M et al (2006). A complex polymorphic region in the brain-derived neurotrophic factor (BDNF) gene confers susceptibility to bipolar disorder and affects transcriptional activity. Mol Psychiatry 11: 695-703.

Otsubo T, Tanaka K, Koda R, Shinoda J, Sano N, Tanaka S et al (2005). Reliability and validity of Japanese version of the Mini-International Neuropsychiatric Interview. Psychiatry Clin Neurosci 59: 517-526.

Robinson LJ, Thompson JM, Gallagher P, Goswami U, Young AH, Ferrier IN et al (2006). A meta-analysis of cognitive deficits in euthymic patients with bipolar disorder. J Affect Disord 93: 105-115.

Sairanen M, O’Leary OF, Knuuttila JE, Castrén E (2007). Chronic antidepressant treatment selectively increases expression of plasticity-related proteins in the hippocampus and medial prefrontal cortex of the rat. Neuroscience 144: 368-374.

Schretlen DJ, Cascella NG, Meyer SM, Kingery LR, Testa SM, Munro CA et al (2007). Neuropsychological functioning in bipolar disorder and schizophrenia. Biol Psychiatry 62: 179-186. 
Sharma T, Antonova L (2003). Cognitive function in schizophrenia. Deficits, functional consequences, and future treatment. Psychiatr Clin North Am 26: 25-40.

Sheehan DV, Lecrubier Y, Sheehan KH, Amorim P, Janavs J, Weiller $\mathrm{E}$ et al (1998). The mini-international neuropsychiatric interview (M.I.N.I.): the development and validation of a structured diagnostic psychiatric interview for DSM-IV and ICD-10. J Clin Psychiatry 59(Suppl 20): 22-57.

Shih RA, Belmonte PL, Zandi PP (2004). A review of the evidence from family, twin and adoption studies for a genetic contribution to adult psychiatric disorders. Int Rev Psychiatry 16: 260-283.

Shinagawa F, Kobayashi S, Fujita K, Maekawa H (1990). Japanese Wechsler Adult Intelligence Scale-Revised. Nihonbunkakagakusha: Tokyo. (in Japanese).

Sugishita M (2001). Japanese Wechsler Memory Scale-Revised. Nihonbunkakagakusha: Tokyo. (in Japanese).
Tadokoro K, Hashimoto R, Tatsumi M, Kamijima K, Kunugi H (2004). Analysis of enhancer activity of a dinucleotide repeat polymorphism in the neurotrophin-3 gene and its association with bipolar disorder. Neuropsychobiology 50: 206-210.

Tamura H, Ishikawa Y, Hino N, Maeda M, Yoshida S, Kaku S et al (2006). Neuropsin is essential for early processes of memory acquisition and Schaffer collateral long-term potentiation in adult mouse hippocampus in vivo. J Physiol 570: 541-551.

Wechsler D (1981). Wechsler Adult Intelligence Scale, Revised. Psychological Corporation: New York.

Wechsler D (1987). Wechsler Memory Scale Manual, Revised. Psychological Corporation: San Antonio.

Yoshida S, Taniguchi M, Hirata A, Shiosaka S (1998). Sequence analysis and expression of human neuropsin cDNA and gene. Gene 213: 9-16.

Supplementary Information accompanies the paper on the Neuropsychopharmacology website (http://www.nature.com/npp) 